\title{
Generalized Streak Lines: Analysis and Visualization of Boundary Induced Vortices
}

\author{
Alexander Wiebel, Xavier Tricoche, Member, IEEE CS, Dominic Schneider, \\ Heike Jänicke and Gerik Scheuermann, Member, IEEE CS
}

\begin{abstract}
We present a method to extract and visualize vortices that originate from bounding walls of three-dimensional timedependent flows. These vortices can be detected using their footprint on the boundary, which consists of critical points in the wall shear stress vector field. In order to follow these critical points and detect their transformations, affected regions of the surface are parameterized. Thus, an existing singularity tracking algorithm devised for planar settings can be applied. The trajectories of the singularities are used as a basis for seeding particles. This leads to a new type of streak line visualization, in which particles are released from a moving source. These generalized streak lines visualize the particles that are ejected from the wall. We demonstrate the usefulness of our method on several transient fluid flow datasets from computational fluid dynamics simulations.
\end{abstract}

Index Terms-Skin friction, singularity tracking, vortex, generalized streak line, flow visualization, time-dependent vector fields.

\section{INTRODUCTION}

Simulation and visualization of fluid flows plays an important role during the design process in engineering practice. Automobiles, turbines, motors, and buildings are only few examples. In a broad variety of applications the onset and behavior of vortices as well as their interaction with the object under consideration is of great practical significance. For automobiles, vortices have an important influence on the drag coefficient, which affects fuel efficiency and overall vehicle performance. In turbines and motors, vortices are critical as they hamper energetic efficiency, while they impact the durability of tall buildings in urban design. In this context, visualization can help to find, analyze and interpret these vortices in the numerical simulations that are used for their study. Typically, the analysis focuses on the three-dimensional structures present in the flow and on their interaction with the shear stress vector field. The latter resembles the tangential flow near the surface of an object and forms the patterns that can be observed in wind tunnel experiments. Vortices, when they interact with the body, leave a certain characteristic footprint in the shear stress field. Vortices tangential to the object boundary drag flow away from the surface along lines of strongly hyperbolic skin friction lines known as separation lines [12]. In contrast, vortices with a core line normal to the surface (see e.g. Fig. 5 right image) or with a certain angle of inclination leave singularities at the locations where their vortex core line touches the object. It has been shown that the topological structure and in particular the singularities of the wall shear stress are essential to characterize the three-dimensional flow structures surrounding the body $[2,26,27]$. A combined structural analysis of the fluid flow and the shear stress vector field thus improves the understanding and interpretation of the phenomena of interest.

In this paper, new methods are presented to track singularities over curved surfaces in time-dependent vector fields and elucidate the interconnections between the critical points of the wall shear stress and the three-dimensional flow. In order to employ efficient singularity tracking schemes for planar settings, selected regions of the surface

- Alexander Wiebel, Dominic Schneider, Heike Jänicke and Gerik Scheuermann are with the Image and Signal Processing Group at the Universiät Leipzig. E-mail:

\{wiebel|schneider|jaenicke|scheuermann\}@informatik.uni-leipzig.de.

- Xavier Tricoche is with SCI Institute, University of Utah, and CS

Department,Purdue University.E-mail:xmt@cs.purdue.edu.

Manuscript received 31 March 2007; accepted 1 August 2007; posted online 2 November 2007.

For information on obtaining reprints of this article, please send e-mail to: tvcg@computer.org. are parameterized and thus mapped to the plane. The paths of the tracked singularities depict the loci, where particles are injected into the three-dimensional flow. These particles form a generalized streak line, which reflects the interrelation between wall shear stress and three-dimensional flow.

The remainder of the paper is organized as follows. We first review previous work on topology-based vector field visualization and in particular singularity tracking schemes in transient vector fields. Then, in Section 3, we describe the method we use for singularity tracking on curved surfaces. Section 4 introduces our concept of generalized streak lines and explains how we combine singularity tracking with the new streak line definition. Section 5 introduces the datasets that were used to test our method and Section 6 discusses the results. Finally, conclusions are drawn in Section 7.

\section{Related Work}

The research topics involved in the present work are briefly considered next.

Singularity Tracking. Topology provides a powerful framework to characterize and study the structures of fluid flows. It has proven successful in scientific visualization and many methods have been proposed $[24,14]$ that leverage its theoretical foundations to achieve effective depictions of complex vector fields. Of particular relevance for the present work are the techniques that permit to track the continuous evolution of the topology as it evolves over time. Improving on a scheme introduced by Helman and Hesselink [11], which graphically reconnects the topological skeletons extracted in successive time steps, Tricoche et al. [30] proposed a scheme that computes the continuous path followed by two-dimensional singularities (where the flow velocity vanishes) across the space-time domain. Their approach explicitly characterizes bifurcations, which correspond to critical changes affecting the structure of the topological skeleton. An alternative method was introduced by Theisel et al. [29], that extracts the topological evolution by means of numerical integration over the space-time continuum. Extensions to three-dimensional transient flows have been presented for both methods $[8,28]$.

In contrast, the tracking of vector field topology over curved surfaces bears specific challenges and has not, to the best of our knowledge, been considered so far in the visualization literature. Related to our work, however, is research that compares flow topology on curved surfaces to the three-dimensional flow, e.g. work by Garth et al. [7].

Vector Field Interpolation Over Polygonal Surfaces. In order to extend existing 2D singularity tracking schemes to polygonal 3D surfaces a proper interpolation scheme must be used that provides a continuous reconstruction of a tangential vector field over the mesh 


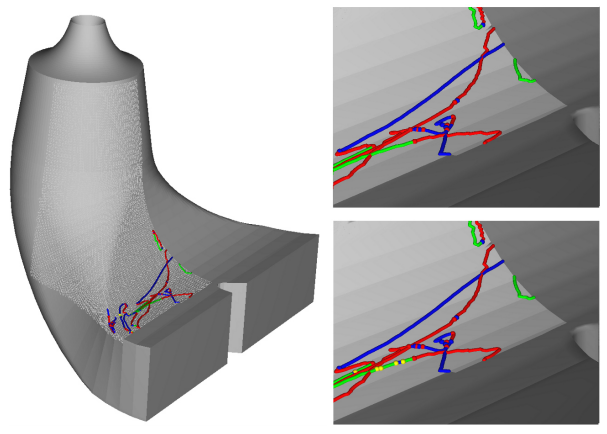

(a) Singularity paths on draft tube.

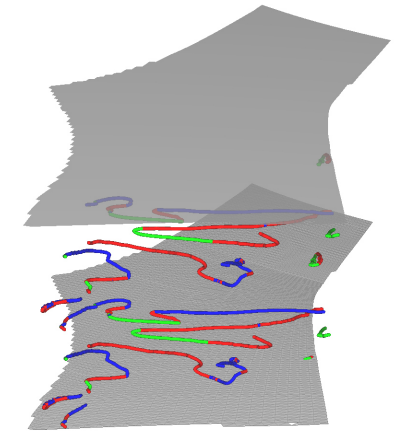

(b) LSCM

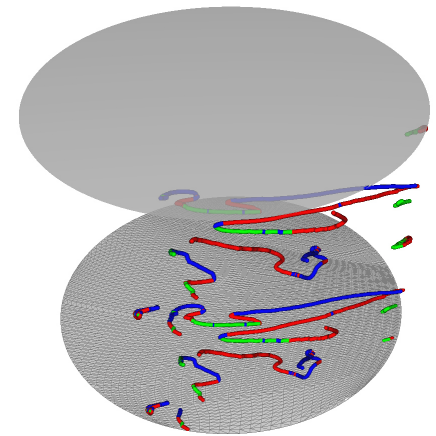

(c) Floater Mean Value Coordinates

Fig. 1. (a) (left) Singularity paths tracked on surface of upper part of draft tube. The region used for tracking is highlighted. The image shows all paths extracted by the tracking method using LSCM parameterization. (right) The upper image shows a close up of the same paths. The lower image shows paths for tracking with mean value parameterization and circular parameter space. The paths computed using LSCM parameterization are smoother, and fewer artificial bifurcations show up. Images (b) and (c) show the singularity paths in in the parameter domain over time.

while being simple enough to permit an efficient and accurate extraction of the corresponding topology. Yet, in the typical setting of a triangle mesh the tangent space is constant within each cell but it is discontinuous across edges and vertices. Hence, the piecewise linear interpolation typically applied over triangle meshes yields discontinuous vector values across triangle boundaries. This leads to spurious topological features when standard extraction algorithms are applied to wall vector fields.

Several approaches have been recently introduced in the computer graphics literature to address this problem. Li et al. [17] proposed a method that models higher-order singularities and visualizes the resulting flow on piecewise-linear surfaces. The authors introduced an interpolation scheme on surfaces that exploits a facet-based representation of vector fields to permit a piecewise $2 \mathrm{D}$ treatment of singularities. However, a feature of this approach is that singularities are constrained to lie on the vertices of the mesh, which makes it unsuitable for our problem. A second approach presented by Zhang et al. [33] defines an interpolation scheme for vector field design over surfaces using geodesic polar maps and parallel transport. While this scheme supports the design of complex phase portraits, it yields a non-linear vector field inside each triangle. The Jacobian matrix required for topological analysis must therefore be approximated through a local linear fit, which seriously complicates the type of analysis required by our method. Additionally, schemes have been proposed in the graphics community that exploit principles from Discrete Exterior Calculus [10] to create tangent vector fields in the limit of a subdivision process [31], or to permit the interactive editing of a discrete tangential vector field over a triangle mesh [3].

Surface Parameterization A way to circumvent the challenge posed by the construction of a suitable interpolation on a polygonal surface consists in transporting the problem to the planar domain by computing a parameterization of the mesh. This subsequently allows for the straightforward application of 2D methods. However, for the body surfaces typically used in numerical simulations this mapping involves cuts and distortion, which in turn requires caution in the interpretation of the results. Various techniques have been proposed in the graphics literature. A discussion of available methods is clearly beyond the scope of this paper and we refer the interested readers to the existing surveys [5, 6]. Most relevant to the present paper are Floater's Mean Value Coordinates method [4] and Levy et al.'s Least Squares Conformal Map [16]. On a related note, observe that the recently introduced image-based flow visualization approach $[15,32]$ is essentially built upon an interactive parameterization of the visible portion of a mesh through simple projection, which permits the extension of dense visualization techniques designed for planar flows to curved surfaces.

\section{Singularity TRACKING ON SURFACES}

As mentioned previously, computing the path followed by the seeds of our generalized streak lines requires to track the singularities of the shear stress vector field over the triangle mesh that models the object boundary. Yet, the piecewise flat nature of the geometry makes this an ill-posed problem. Indeed, the discontinuity of the tangential vector field across edges implies that a singularity leaving a cell will not, in general, be able to resume its motion from the corresponding location in the next cell. For instance, no singularity might be present on the border or in the interior of the neighboring cell at the same instant, or its type might be incompatible with the one of the currently followed singularity in terms of local topology consistency. In any case, the corresponding discontinuity challenges the very idea of tracking the continuous transformation of the topology.

Following the observations we made in the previous section, a possible way to tackle this problem consists in parameterizing the mesh such that further computation can be carried out in the plane using the algorithms available in this setting. Observe that this transposition is more than a mere computational fix to the tracking problem. Its deeper motivation lies in the fact that the piecewise linear geometry representation used for numerical simulations is itself an approximation of what in reality is a smooth object surface. Using the available discrete information, our goal is therefore to bring the problem back to a setting where surface topology and the continuous transformation thereof are well-defined and applicable concepts, as they are in the physical realm. We provide in this section a description of our tracking algorithm and discuss possible limitations. In particular, to avoid the difficulty of computing a smooth global parameterization of the whole surface, we choose to restrict our analysis to subregions of the triangle mesh. With that assumption, our algorithm consists of 4 main steps.

1. Select an interesting subregion

2. Parameterize subregion in the plane and express vector field in parameter space

3. Perform 2D singularity tracking in parameter space

4. Map nodes of singularity paths back onto the triangle mesh for visualization

We detail each of these steps in the following and proceed with considerations linked to the visualization of the topological information.

\subsection{Region Selection}

As pointed out above, our method is designed to track singularities on subregions of a surface. To be able to compute the parameterization of 

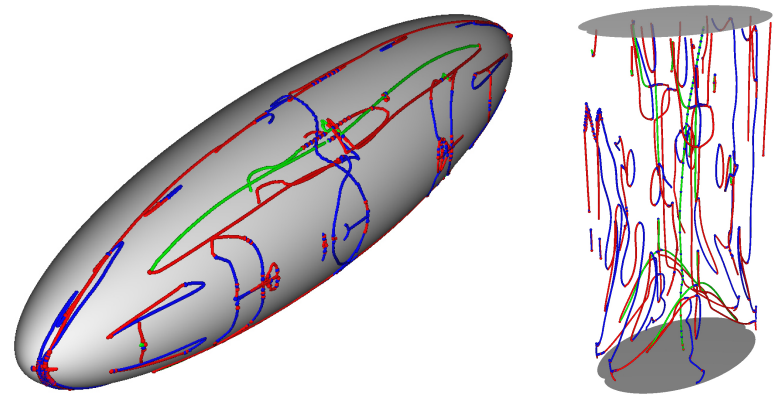

Fig. 2. Singularity paths on the surface of ellipsoid tracked using LSCM parameterization. left: Paths for all time steps on surface of ellipsoid. right: Paths in parameter domain with time as third dimension.

a region our current implementation imposes some restrictions. First, we require the region to be homeomorphic to a disc. Second, assuming an a priori knowledge about the area in which a particular singularity exists, we choose the region such that the singularity stays sufficiently far to the region boundary over the whole course of its motion. Third, we impose that the region does not contain sharp edges of the geometry. This last requirement is primarily motivated by the fact that sharp edges in the design will typically create natural flow discontinuities which the parameterization should not attempt to artificially smooth out.

Note that because our implementation uses parameterization algorithms designed for regions homeomorphic to a disc, we impose this criterion to the regions that we select. Hence, we are not currently able to track singularities that would travel over more complex surface regions. This is not an intrinsic limitation of our approach, however, since parameterization plays the role of a black box in our method and more advanced, smooth global parameterization techniques exist (e.g. $[13,21])$ that could be used instead. See also the corresponding discussion in Section 7.

\subsection{Parameterization}

To map the shear stress vector field to a $2 \mathrm{D}$ vector field we compute a parameterization of the surface subregion under consideration. For that purpose we have experimented with the Floater Mean Value Coordinates parameterization method [4] and the Least Squares Conformal Map (LSCM) [16], both of which are provided by the geometric library CGAL [22]. The shape of the parameter space is a circle for the mean value parameterization and a free boundary in the case of LSCM. The LSCM parameterization produces less distorted triangles in the parameter space and thus a better parameterization. Its drawback, however, is that the mapping cannot be guaranteed to be oneto-one (in contrast to mean value coordinates) although it typically is $[5,6]$. For planar regions in $3 \mathrm{D}$ we obviously use the canonical (isometric) parameterization obtained by simply defining a local coordinate system directly on the surface.

Once the considered region has been parameterized, its corresponding (time-dependent) vector values must be transformed accordingly. Obtaining 2D coordinates for those vectors amounts to projecting each $3 \mathrm{D}$ vector attached to a vertex of the mesh onto the basis vectors of the local tangent plane induced by its local parameterization. Specifically, with $\mathbf{f}: U \subset \mathbb{R}^{2} \rightarrow S \subset \mathbb{R}^{3}$ denoting the function mapping the 2D parameter domain to the surface $S$ and $\mathbf{p} \in U, \mathbf{Q} \in S$ such that $\mathbf{f}(\mathbf{p})=\mathbf{Q}$, the vector $\vec{v}_{\mathbf{p}}$ in parameter space corresponding to $\vec{V}_{\mathbf{Q}}$ in $3 \mathrm{D}$ has the 2D coordinates $\vec{v}_{\mathbf{p}}=\left(\left.\vec{V}_{\mathbf{Q}} \cdot \frac{\partial \mathbf{f}}{\partial u}\right|_{\mathbf{p}},\left.\vec{V}_{\mathbf{Q}} \cdot \frac{\partial \mathbf{f}}{\partial v}\right|_{\mathbf{p}}\right)$.

\subsection{Singularity Tracking in the Plane}

To track the singularities of the resulting transient planar vector field we use the technique proposed by Tricoche et al. [30]. Alternatively, the same results can be achieved with the Feature Flow Field approach of Theisel et al. [29]. In a nutshell, both schemes follow the trajectories described by the singularities over a continuous reconstruction of the space-time continuum and, along the way, detect bifurcations that cause the creation and annihilation of singularities, as well as their type change. Refer to the original papers for additional details. The 2D-tracking provides us with a polygonal description of the singularity paths in the parameter space, along with associated bifurcations. This information can then be mapped back in the physical space using the parameterization function and its linear nature inside each triangle.

It is important to note that the reformulation of the tracking problem in the parameterization domain can generate artifacts in the form of spurious topological features that have no practical significance. In our case however, we choose the regions in such a way that these artifacts should be minimized. More importantly, because of the ill-posed character of the tracking problem in the first place, we do not have a ground truth with which to compare our results. Therefore we adopt a more pragmatic approach. First, we apply filtering criteria to the resulting singularity paths that are designed to prune insignificant topological features to which we expect artifacts to belong. Second, the validation of our singularity paths comes from their use as seed points for the generalized streak lines described below. Ultimately our results are deemed valid if they show good correlation with the transient three-dimensional flow structure.

\subsection{Visualization}

Visualizing the paths of singularities moving on surfaces is not a trivial task. There are at least three types of information that are necessary to allow a scientist to interpret the paths: positions of nodes, type of the singularity represented by a path and the time the singularity is located at a certain position. If the paths are simply drawn as lines on the surface, all information about their temporal extent and distribution is lost, which makes it very hard to interpret the resulting images. Incorporating temporal information in the line visualization by encoding the advancing time as changing the color is not feasible because color is rather used to describe the type of the singularity.

For 2D time-dependent fields the third dimension is used to illustrate the advancing time. This yields $3 \mathrm{D}$ representations of the changing topology. Unfortunately, it is not possible to apply this approach directly to time-dependent vector fields on surfaces as these are of three-dimensional nature themselves.

Instead, we decided to apply the standard 2D visualization approach to the two-dimensional space we use for the tracking. This results in a more schematic representation of the developing topology (see Figs. 1, 2 and 7). Although it does not reflect the geometry of the surface, we believe that this schematic representation in combination with drawing the paths on the surface, achieves the best overview possible with a static visualization. Allowing the user to mark regions in the schematic representation and highlighting the corresponding parts of the paths on the surface in a focus and context fashion improves the utility of this method (Figs. 7(b), 7(f)). In the images of Fig. 7 we additionally emphasized the paths of the sinks, as these are important for the generalized streak lines. In combination with filtering out paths of short-lived and thus insignificant singularities, this results in much less cluttered and more insightful images (compare Fig. 7(a) to 7(c)-7(d)).

All figures (except Figs. 7(b)-7(d),7(f)) showing singularity paths use the following color scheme. Paths of saddles are drawn as red lines, paths of sinks (attracting nodes) are drawn as blue lines and paths of sources (repelling nodes) are drawn as green lines. Bifurcations are represented by spheres. Red spheres indicate creation of singularities, blue spheres annihilation of singularities and yellow spheres indicate Hopf bifurcations.

\section{Generalized Streak Line}

In this section, we introduce a generalization of streak lines. For this purpose, we review the mathematical definitions and interpretations of streak lines and path lines. Let $\mathbf{v}: \mathbb{R}^{3} \times U \subset \mathbb{R} \rightarrow \mathbb{R}^{3}$ be a Lipschitz continuous time-dependent vector field. Let $\mathbf{a} \in \mathbb{R}^{3}$ be the position of a particle in space and let $t \in U$ be a certain time. We begin with path lines, as streak lines can be expressed in a very simple way using path lines. 

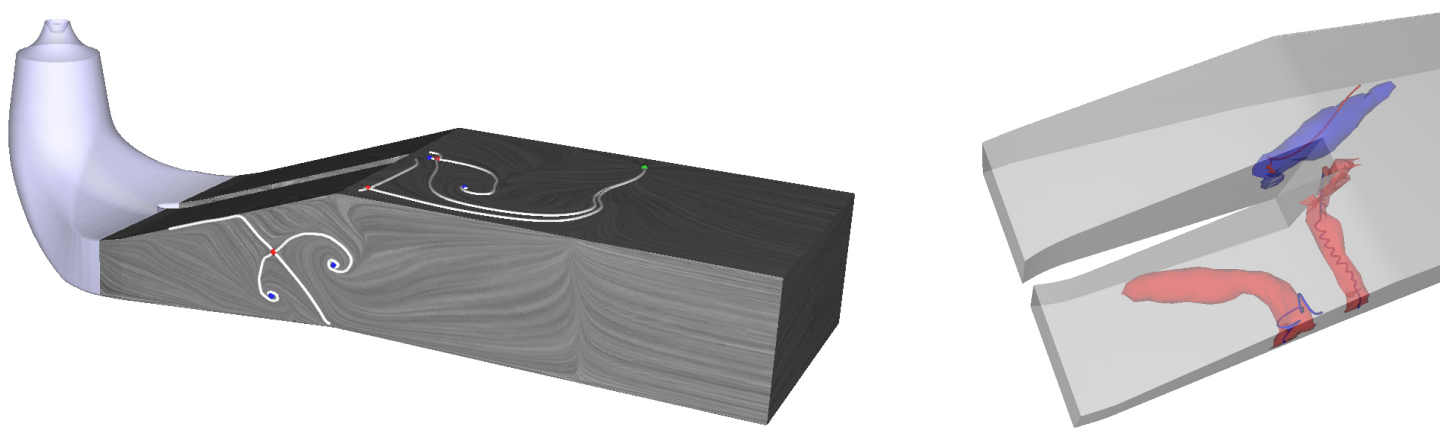

Fig. 3. Overview of draft tube dataset. left: Parts of the flow topology on the surface of the lower part of the turbine draft tube. Sinks (blue) and separatrices (white lines) on top of LIC texture indicate the existence of three vortices on the top and the right side of the tube. right: Isosurfaces of $\lambda_{2}$ seeded near singularities on side wall show vortices causing the singularities on the wall. Streamlines show the vortical behavior.

Path lines are integral curves $\mathbf{p}_{\mathbf{a}, t_{0}}(t)$ of time-dependent vector fields, which are tangential to the vectors of a field's domain. They are the trajectories of massless particles moving in a flow defined by the vector field. Mathematically, this reads as follows:

$$
\begin{aligned}
t & \mapsto \mathbf{p}_{\mathbf{a}, t_{0}}(t) \\
\mathbf{p}_{\mathbf{a}, t_{0}}\left(t_{0}\right) & =\mathbf{a} \\
\frac{\partial \mathbf{p}_{\mathbf{a}, t_{0}}}{\partial t}(t) & =\mathbf{v}\left(\mathbf{p}_{\mathbf{a}, t_{0}}(t), t\right),
\end{aligned}
$$

where $t_{0}$ is the seed time.

Streak lines $\mathbf{l}_{\mathbf{a}, t}$ are imaginary lines connecting the locations of particles that were released into a flow from a certain location at consecutive time steps. Thus, when dye or some other marking material is discharged slowly at some fixed point in a moving fluid, the visible line produced in the fluid is a streak line (see e.g. Batchelor [1], Lugt [18]). The lines can be observed when looking at the particles at a certain time $t$.

$$
s \quad \mapsto \quad \mathbf{l}_{\mathbf{a}, t}(s)=\mathbf{p}_{\mathbf{a}, s}(t)
$$

Note that $t$ is fixed and $s$ is the varying seed time.

Although we have not found it in the literature, our generalization of streak lines is straight forward. Instead of releasing particles from a stationary source, we consider particles released from a moving source. Thus, generalized streak lines $\mathbf{1}_{\mathbf{c}, t}$ are defined as imaginary lines connecting the locations of particles that were released into a flow from a location $\mathbf{a}(s)$ continuously moving along a path $\mathbf{c}$ at consecutive time steps. The line can be observed when looking at the particles at a certain time $t$.

$$
s \mapsto \mathbf{l}_{\mathbf{c}, t}(s)=\mathbf{p}_{\mathbf{a}(s), s}(t)
$$

Again $t$ is fixed and $s$ varies.

As far as we know, this type of streak lines has not been treated in the visualization literature yet. This may be the case, as in experiments the creation of streak lines from moving sources is not a simple task. It becomes even harder, when trying not to influence the flow by the movement of the source.

\subsection{Singularity Streak Lines}

The generalized streak lines considered throughout this paper use the locations of moving singularities as locations of the particle source (particle injection points). We call these special streak lines singularity streak lines. While moving sources in general may be achievable for experiments, moving sources with singularities is impossible. One would have to detect the singularities in the experiment and at the same time move the source accordingly. This is not possible. We will discuss the importance and meaning of streak lines starting from singularities in the following.

\subsubsection{Singularity Paths as Particle Source}

As mentioned earlier, vortices in the three-dimensional flow leave singularities of the shear stress field as footprints on a body's surface. The singularities are spiral sources if the flow attaches to the surface and spiral sinks if flow leaves the surface, i.e. when particles are ejected from the surface into the volume. Indeed, all singularities on the body surface correspond to half-saddles in the three-dimensional flow around the body. The 2D flow around the singularities on the body are the unstable (sources) or stable (sinks) 2D-manifolds of the threedimensional saddles, i.e. the manifolds coincide with the body surface. The remaining separatrix resides in the three-dimensional flow. It is a stable manifold for sources and an unstable manifold for sinks on the surface.

The unstable manifold in the three-dimensional flow is the reason why we will consider only spiral sinks here. Our aim is to elucidate which parts of the domain are reached by particles that leave the surface through the vortex, or better where these particles are at a certain time. This gives information about which regions of the flow are influenced by the vortex corresponding to the sinks. It is also interesting, when considering vorticity transport, as some of the vorticity which is present at a certain position is advected ${ }^{1}$ by particles passing the position, and thus is transferred to other regions of space. This is especially important since for incompressible flows vorticity can only be generated at walls or enter the fluid from an open boundary [1]. In other words, it cannot be generated inside the fluid. This does not mean that vortices cannot develop in the flow, which actually may be the case, when vorticity concentrates at some point in space. It means that the vorticity has either to be present in the flow right from the beginning of observation time or that vorticity is advected or diffused into the flow from the boundary.

Motivated by the aims mentioned in the previous paragraph, we follow the particles ejected into the flow from a sink on the surface and with them a portion of the vorticity present at the boundary. We use the positions along a singularity path as particle source for the generalized streak line integration. Natural start and stop positions for the particle injection are given by the bifurcations bounding a singularity path as a singularity is created, destroyed or changes its type there. Beginning at a creation point or a Hopf bifurcation we move along the line in time and space, and release particles until we reach the next bifurcation where we stop releasing particles. However, this does not mean that the streak line stops its evolution. We continue tracking the present particles. As the particles move away from their initial position, the streak line separates from the singularity path. After separation the streak line still consists only of particles that originate from the singularity on the surface.

\footnotetext{
${ }^{1}$ Visualization techniques for vorticity transport (diffusion and advection) where presented by Sadlo et al. [23].
} 


\subsubsection{Issues Concerning Starting Particles Near Singularities}

We mentioned above that the singularities on the surface correspond to $3 \mathrm{D}$ half-saddles in the volume. It is well known that starting streamline integration at saddle points in order to obtain separatrices is problematic. It is a standard problem that has to be handled by any implementation trying to extract the topological graph of a steady vector field. It is not possible to start the streamline exactly at the position of the saddle as the velocity is zero there. All implementations have to take a small step away from the saddle to obtain non-singular starting points for separatrices.

The singularities we treat have a two-fold nature. They live on the surface as 2D sinks and in 3D as half-saddles. We have to take a small step along the unstable manifold living in 3D. A first very simple approximation is to take the step normal to the surface. Small numerical inaccuracies introduced by stepping away along the normal vector and not along the actual unstable manifold, are compensated by the strong hyperbolicity of the flow along the manifold. All streamlines around the unstable manifold converge to it. Another possibility is to extract a singularity of the projected flow on an offset surface having a distance of one cell layer from the actual surface. The singularity in the projected flow of this layer is very close to the original singularity. Only the projected flow vanishes there, the original $3 \mathrm{D}$ vector at the position of the singularity can be used to start a particle's motion.

The correct direction is given by the eigenvector corresponding to the largest real eigenvector of the Jacobian of the 3D singularity. To be able to use this vector the $3 \mathrm{D}$ singularity belonging to the $2 \mathrm{D}$ sink has to be identified. This is possible using an interpolation scheme for incompressible flow proposed by Peikert et al. [20].

As we found it to be sufficiently accurate, our implementation uses the normal vector direction for the step.

\section{Datasets}

We present results of applying our methods to four different CFD datasets in Section 6. The datasets are described shortly in this section.

\subsection{BMW}

The BMW dataset stems from a steady simulation around the right half of the car while assuming a flow symmetry plane along the middle of the car. Fig. 4 gives an overview of some of the important features of the dataset. The red lines represent vortex core lines extracted by the algorithm of Sujudi and Haimes [25] in the parallel vectors version of Roth and Peikert [19]. The strongest vortices appear behind the car, the tires, the side mirror and at the windscreen. We will go into detail about the relevance of this steady dataset in the results section.

\subsection{Draft Tube}

This dataset represents the draft tube of a Francis turbine, in which the runner is spinning in the inlet part of the turbine (see Fig. 3, upper left corner of left image). The runner induces a spinning motion in the water, which leaves the turbine (right part of left image) after passing through the tube. The inlet of the lower part of the tube is split into two channels. The upper part is dominated by one main vortex, while the flow develops several distinguished vortices that are connected to the boundary in the lower part. There are 300 time steps representing 0.4 seconds of physical time available to us.

\subsection{Cuboid}

The cuboid dataset results from direct numerical simulation of fluid flow around a cuboid at a Reynolds number of $R e=1000$. The simulation was carried out with the NaSt3DGP ${ }^{2}$ flow solver. A version of the NaSt3DGP code, as well as related information and documentation is available for download at http: //wissrech.iam. uni-bonn. de/research/projects/NaSt 3DGP/index.htm. We used a slightly modified version of the flow past an obstacle example, which

${ }^{2}$ NaSt3DGP was developed by the research group in the Division of Scientific Computing and Numerical Simulation at the University of Bonn. It is essentially based on the code described in a book by Griebel et al. [9].
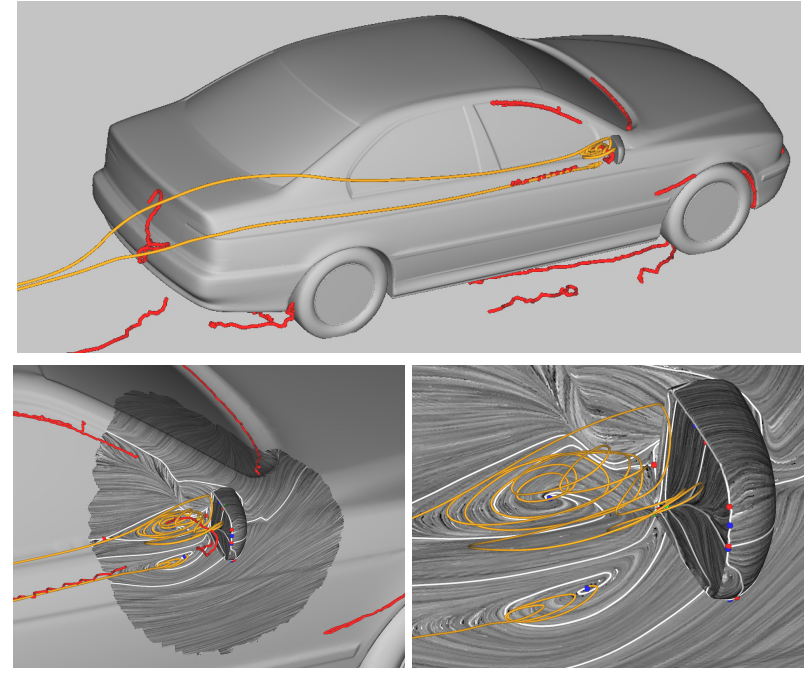

Fig. 4. Visualization of important vortices around BMW car. top: Overview of dataset showing car with streamlines started near right side mirror and vortex core lines. left: Close up with LIC and topology graph on surface near side mirror. The swirling behavior of the streamlines nicely correlates with the vortex core lines. right: Streamlines start directly from singularities in surface shear stress field.

is also available on the web site. The flow enters the simulation region with a velocity of $\sqrt{8} \frac{\mathrm{m}}{\mathrm{s}}$. The velocity data is stored as a vector field on a $100 \times 100 \times 100$ rectilinear grid. We use 1355 time steps that represent 100 seconds of physical time. As can be seen by examining the stream surface in Fig. 5, the flow behavior behind the cuboid is quite chaotic. It is dominated by a large number of vortices that originate from the edges and faces of the cuboid. The LIC texture and the topology graph on the surface of the cuboid (Fig. 5 middle) show the footprints of separation structures and the mentioned vortices. The image on the right (Fig. 5) shows the connection between a vortex and its corresponding attracting spiral node by some streamlines started near the node. The streamlines lead away from the surface as the vortex drags particles away from the cuboid.

\subsection{Ellipsoid}

A flow around an ellipsoid at Reynolds number $R e=10000$ was simulated to obtain this dataset. We use every tenth of the computed time steps resulting in a total number of 600 steps capturing 3 seconds of physical time. The surface of the ellipsoid is a triangular grid which has a cut at the front side shown in the left image of Fig 2. Thus the surface is homeomorphic to a disc and ready for the application of our parameterization. The flow around the ellipsoid develops two main vortices in the beginning (see Fig. 9 left image). These vortices interact and evolve into a more complex yet symmetric pattern. The main rotation axis of the vortices in later time steps is orthogonal to the axis of the early vortices. This is nicely depicted by the streak line in last two images of Fig. 9. We will discuss the evolution of the singularity streak line in the results section.

\section{Results}

The aim of our method is to analyze the interaction of the wall shear stress with the three-dimensional flow. Especially we are interested in singularities belonging to vortices originating from the wall and the vorticity transported by the particles ejected from the wall at the singularities. This is accomplished in three steps: surface parameterization, singularities tracking, and visualization of generalized streak lines and related structures. The results of the individual steps are explained in the following. 

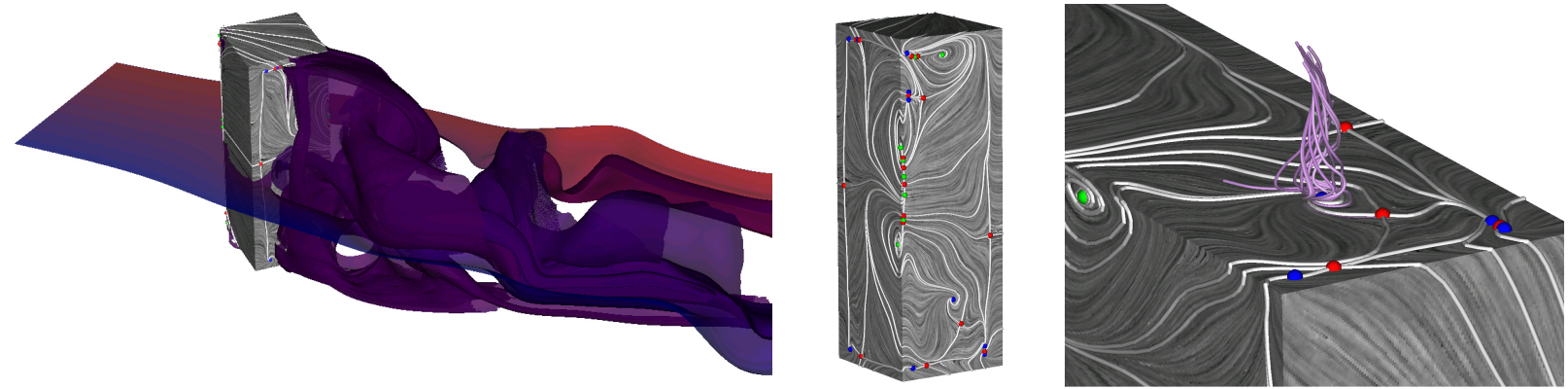

Fig. 5. Depictions of the flow in the cuboid dataset after 50 seconds of physical time. left: Stream surface showing turbulent behavior behind cuboid. middle: LIC and topological structures of shear stress field on cuboid. right: Swirling streamlines indicating existence of vortex above shear stress field sink (sink is located in lower right part of middle image).

\subsection{Parameterization}

In order to apply efficient tracking algorithms for planar settings the surface is parameterized and thus can be mapped to the plane. The 2D-mappings of the datasets are visualized in Figs. 1, 2(right) and 7. For the draft tube the subregion highlighted as triangulated grid in Fig. 1(a) is parameterized using mean value and LSCM parameterization. LSCM has a free boundary, whereby the relation to the 3Dpatch is clearly visible. As explained in Section 3.2, LSCM parameterization is sometimes not applicable and the mean value parameterization has to be used, which maps the subregion to a disk. Despite the potential confusion introduced by the parameterization procedure which can substantially deform the considered spatial region (see Section 3.2), visualizing singularity paths in the parameter space allows for an effective user interaction when combined with the simultaneous depiction of the selected information directly on the surface.

\subsection{Singularities Tracking}

After the surface is parameterized, 2D singularity tracks can be computed. As explained in Section 3.4, the visualization of the trajectories of the singularities directly on the 3D surface can be rather confusing. Thus, we shift the visualization to the $2 \mathrm{D}$ parameter space. The spatial location is given in $2 \mathrm{D}$ and the third dimension is used to encode time, as visualized in Figs. 1(right), 2(right), and 7.

Moreover, the visualization in parameter space gives a good overview over the dynamics of the flow. The turbulent behavior of the cuboid dataset is clearly reflected in the complex topological structure and its evolution (Fig. 7(e)). Before the turbulence develops, the singularity paths exhibit a clear, nearly symmetric shape. A large number of singularities appear suddenly when the flow develops the turbulent behavior. These effects decay after the flow reaches a certain amount of turbulence. Displaying the paths of the upper part of the draft tube in the parameter space (Fig. 1) nicely shows the quasi periodic behavior of the rotating flow as a repeating pattern in the singularity paths (two layers with the same pattern).

As can be seen in the cuboid example (Fig. 7), the temporal structure of the trajectories can become very complex, hampering the selection of interesting singularities. We use two different techniques to enhance the visualization. First, an advantageous color coding can be used. When looking for boundary induced vortices, sink paths are of special interest as explained in Section 4.1. The corresponding trajectories are highlighted in a dominant color, while all other singularity paths are displayed as smaller tubes in a paler color to provide context. If only a certain range of time-steps is of interest, color coding is employed to highlight temporal intervals (Fig. 7(b)). Second, filtering can be used to extract singularity paths fulfilling certain properties, e.g., the singularities are located in a certain region, or as in our case, the trajectories are present for a minimal amount of time. Thus, the number of trajectories is significantly reduced, simplifying the selection of relevant singularities (compare Figs. 7(c) and 7(d)). Additionally, it can be useful to show the strength of the vortical behavior along the paths.
When interpreting the tracking results, attention has to be payed to the choice of parameterization. In Fig. 1(top/left) the resulting tracks of mean value and LSCM parameterization are displayed. Insignificant, spontaneous changes of the singularity type appear in the middle image along the path of the repelling node in the lower part of the image. Note that this does not mean appearance or disappearance of singularities. These changes are due to the deformations induced by the mean value parameterization, and can be prevented using the LSCM parameterization, which produces smoother results.

\subsection{Generalized Streak Lines and Vortices}

\subsubsection{Steady Vector Fields}

We discuss the steady BMW dataset here, because it nicely shows the connection between the singularities on the surface and the vortex generation in a common real world setting. For a steady vector field there is no difference between streamlines, path lines and streak lines. Thus the streamlines shown in the images are identical to streak lines. Moreover, the streamlines are identical to the singularity streak lines as singularities do not move in the steady case. The streamlines, thus, identify regions that are influenced by the particles emanating from the singularity on the surface and the corresponding vortex. The vorticity transported with particles is distributed all along the streamlines and contributes to the persistence of the vortical behavior or even to the creation of new vortices in the neighborhood of the streamlines.

Our discussion will focus on the region around the side mirror as it is quite obvious that vortices develop behind such a protruding part of the geometry. The lower images of Fig. 4 show a LIC texture and the topology graph (white lines) of the surface flow near the side mirror. The orange streamlines originating from the spiral sinks swirl around the vortex cores lines. The vortex core line belonging to the lower of the two vortices is not captured completely by the extraction method. However, at some distance from the surface the streamline nicely resembles the vortex core line.
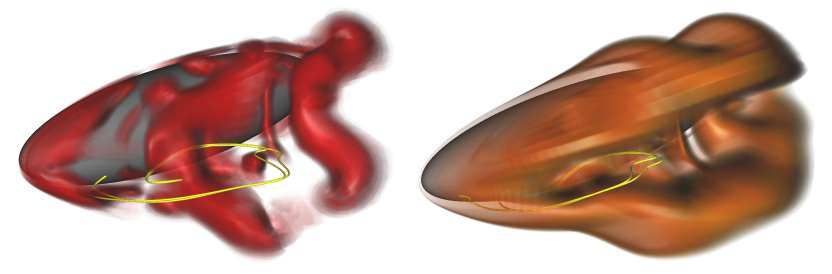

Fig. 6. Ellipsoid dataset: singularity streak line with volume rendering of $\lambda_{2}$-criterion (left) and vorticity (right).

\subsubsection{Unsteady Vector Fields}

In the unsteady case, a singularity streak line can be computed and visualized after selecting an interesting singularity path. The images 
in Fig. 8 are taken from an animation ${ }^{3}$ of the cuboid dataset that shows the evolution of the particles emitted from one of the attracting spiral nodes on the surface. The images show (from left to right) how the starting position of the streak line moves along the singularity path (turquoise). In the third image the singularity has become a source (repelling) through a Hopf bifurcation. Thus, it does not emit any particles into the 3D flow anymore. We stop particle injection at this point and allow the streak line to separate from the surface (see last image). The images cover only a relatively short period of time (24.5 seconds) but already show a large amount of winding and bending of the streak line, which is due to the turbulent behavior behind the cuboid.

Additionally, the streak line experiences strong stretching over time. It covers nearly the complete region behind the cuboid in later time steps. We do not show an image of such a time step as it strongly suffers from clutter. However, after careful investigation it turns out that the streak line forms coherent patterns. One can observe the shape of hairpin vortices in these patterns. In fact, comparison with isosurfaces of $\lambda_{2}$ shows that hairpin vortices evolve behind the cuboid and are fully developed shortly before the flow leaves the simulation domain. It is quite intelligible that the particles of the streak line agglomerate in a vortex as they transport vorticity and a vorticity concentration is one of the characteristics of a vortex.

Images from an animation ${ }^{3}$ of our second singularity streak line example, the ellipsoid, are shown in Fig. 9. The origin of the streak line, i.e. the sink path in the lower left part of the images (blue), lies in an area of low pressure (first two images). The streak line winds around a region of low pressure in the fourth and fifth image. Low pressure can serve as vortex indicator in many cases. Indeed the regions of low pressure in our dataset are co-located with vortices. Fig. 6 illustrates the vortices by volume rendering of $\lambda_{2}$ and vorticity in comparison to the streak line.

\subsection{Performance}

We list the computation time for performing the complete tracking procedure on our datasets in Table 1. This includes transforming the vector into parameter space, tracking, sorting of the line segments to tracks, sorting the tracks by arclength and displaying all paths on the surface. The computations were carried out on one core of an AMD Opteron $2210(1.8 \mathrm{GHz})$ with $8 \mathrm{~GB}$ main memory. Our software is written in $\mathrm{C}++$ and runs on Linux.

The time cost of the tracking depends on the number of time steps, the number of singularities and the number of cells. The number of singularities is of special importance as they are separately tracked through the time steps of the dataset.

\section{CONCLUSION AND OUtLOOK}

We have presented methods for singularity tracking on surfaces and visualization of vortices that originate from walls in three-dimensional time-dependent vector fields. As such vortices leave singularities as footprint in the wall shear stress vector field, we combined the singularity tracking with a generalized type of streak lines by using the singularity paths as start positions for the particles of the streak lines. These streak lines show the region of the flow which is influenced by the vortex belonging to the singularity. Displaying the particles ejected from the boundary helps illustrating the transport of the vorticity these particles advect from the boundary. This is important because for incompressible flows vorticity is only created at the boundary and is a fundamental quantity in the dynamics of vortices. Applied to several CFD datasets the presented methods proved to be applicable and robust. In accordance with the literature cited in the introduction, fluid dynamicists told us that they are specially interested in the particles leaving the surface. Singularity streak lines are of special interest as they can not be reproduced in experiments.

We plan to extend the applicability of our singularity tracking method in a way that it can handle arbitrary surfaces. This may be possible by correctly connecting parameterizations used for tracking

\footnotetext{
${ }^{3}$ See accompanying video.
}

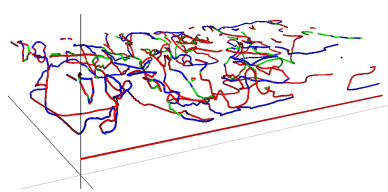

(a)

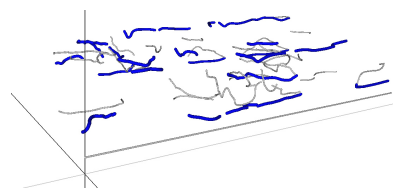

(c)

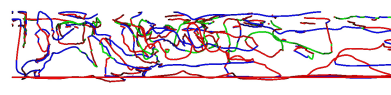

(e)

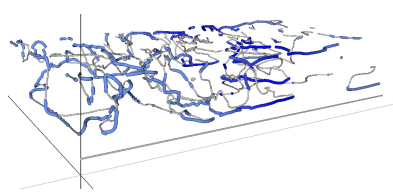

(b)

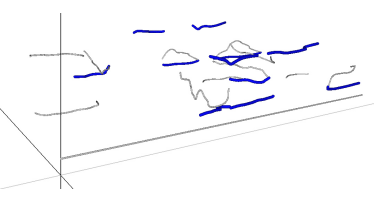

(d)

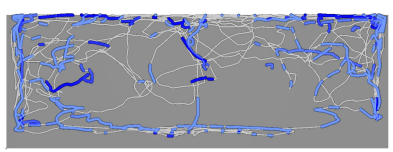

(f)
Fig. 7. Singularity paths for right rear cuboid face. (a) Paths in parameter space. Grey axis indicates time. (b) Sink paths are marked blue and their radius is increased. Other paths in grey. Sink paths in time interval of interest highlighted by dark blue in contrast to light blue. (c) Without highlighting but filtered by length of time of paths. (d) The same with a larger time threshold. (e) Projection along one spatial axis. Time and remaining spatial axis shown. (f) Paths directly on the surface of the cuboid instead of in the parameter space. Interval of interest highlighted.

\begin{tabular}{|l|rrr|}
\hline Dataset & cells & time steps & times (sec) \\
\hline \hline Cuboid side face & 368 & 1355 & 30 \\
Draft tube upper part (Mean) & $8.8 \mathrm{k}$ & 300 & 26 \\
Draft tube upper part (LSCM) & $8.8 \mathrm{k}$ & 300 & 32 \\
Ellipsoid (LSCM) & $39.4 \mathrm{k}$ & 400 & 276 \\
\hline
\end{tabular}

Table 1. Run times for singularity tracking.

in patches that our current method can handle. Atlas-based methods like the ones mentioned in the related work (Section 2) may be helpful here. The visualization of the flow that is ejected from the boundary could benefit from the availability of a good path surface computation method.

\section{ACKNOWLEDGEMENTS}

The authors would like to thank BMW and Markus Rütten from German Aerospace Center (DLR) in Göttingen, as well as VA Tech Hydro and Ronald Peikert from ETH Zürich for providing the data sets. Special thanks for all the fruitful discussions and help go (as always) to Mario Hlawitschka. For helpful answers on some fluid dynamics questions they would like to thank Wolfgang Kollmann and Hassan Aref. Thanks also go to the FAnToM development group. Finally, they wish to thank the anonymous reviewers for their valuable comments and suggestions. This work was partly supported by DFG grant SCHE 663/3-7.

\section{REFERENCES}

[1] G. K. Batchelor. An Introduction to Fluid Dynamics. Cambridge University Press, 1967.

[2] U. Dallmann. Topological Structures of Three-Dimensional Flow Separations. Technical Report 221-82 A 07, Deutsche Forschungs- und Versuchsanstalt fuer Luft- und Raumfahrt, 1983.

[3] M. Fischer, P. Schröder, H. Desbrun, and H. Hoppe. Design of Tangent Vector Fields. SIGGRAPH '07, 2007, to appear.

[4] M. S. Floater. Mean Value Coordinates. Computer Aided Design, 20(1):19-27, 2003

[5] M. S. Floater and K. Hormann. Parameterization of Triangulations and Unorganized Points. In A. Iske, E. Quak, and M. S. Floater, editors, 

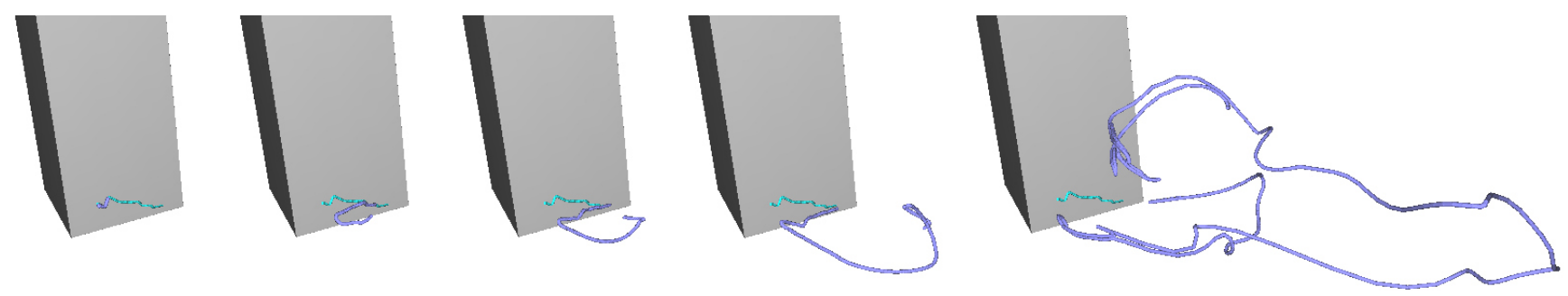

Fig. 8. Generalized streak line in cuboid dataset. The streak line (blue) starts from the moving position of the singularity. The positions, i.e. the path (turquoise), is located in the lower part of the cuboid. After the singularity reaches the Hopf bifurcation at the right end of the path the computation stops inserting new particles for the streak line. Thus the streak line separates from the singularity path (right most image).
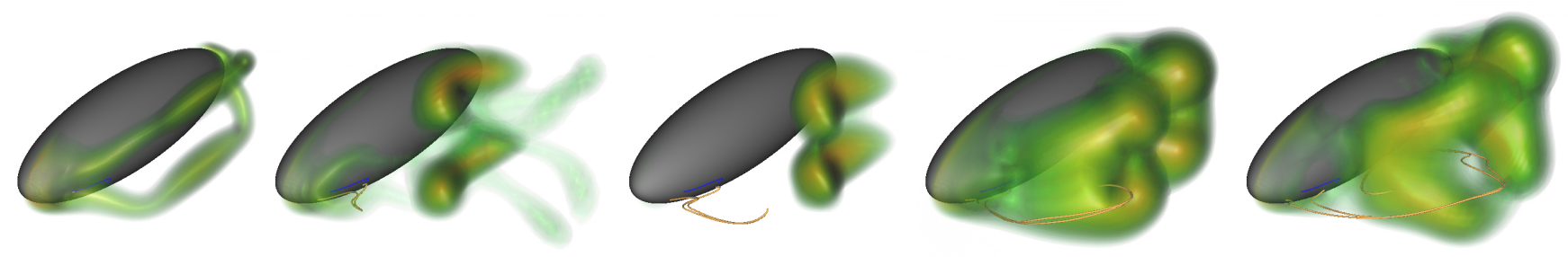

Fig. 9. Snapshots taken from animation ${ }^{3}$ of generalized streak line and volume rendering of pressure in ellipsoid dataset. Low pressure is mapped to high opacity. The generalized streak line starts from blue sink path in the lower left of the images (see e.g. third image).

Tutorials on Multiresolution in Geometric Modelling, Mathematics and Visualization, pages 287-316. Springer, Berlin, Heidelberg, 2002.

[6] M. S. Floater and K. Hormann. Surface Parameterization: A Tutorial and Survey. In N. A. Dodgson, M. S. Floater, and M. A. Sabin, editors, Advances in Multiresolution for Geometric Modelling, Mathematics and Visualization, pages 157-186. Springer, Berlin, Heidelberg, 2005.

[7] C. Garth, R. S. Laramee, X. Tricoche, J. Schneider, and H. Hagen. Extraction and Visualization of Swirl and Tumble Motion from Engine Simulation Data. In Topology-based Methods in Visualization, Mathematics+Visualization. Springer, 2007.

[8] C. Garth, X. Tricoche, and G. Scheuermann. Tracking of Vector Field Singularities in Unstructured 3D Time-Dependent Datasets. In H. Rushmeier, G. Turk, and J. J. van Wijk, editors, Proc. of the IEEE Visualization 2004 (VIS'04), pages 329 - 336. IEEE Computer Society, October 2004.

[9] M. Griebel, T. Dornseifer, and T. Neunhoeffer. Numerical Simulation in Fluid Dynamics, a Practical Introduction. SIAM, Philadelphia, 1998.

[10] E. Grinspun, P. Schröder, and M. Desbrun. Discrete Differential Geometry: An Applied Introduction. In SIGGRAPH'05 Course Notes, 2005.

[11] J. L. Helman and L. Hesselink. Surface Representations of Two- and Three-Dimensional Fluid Flow Topology. In VIS '90: Proceedings of the 1st Conference on Visualization '90, pages 6-13, Los Alamitos, CA, USA, 1990. IEEE Computer Society Press.

[12] D. N. Kenwright, C. Henze, and C. Levit. Features Extraction of Separation and Attachment Lines. IEEE Transactions on Visualization and Computer Graphics, 5(2):135-144, 1999.

[13] A. Khodakovsky, N. Litke, and P. Schröder. Globally Smooth Parameterizations with Low Distortion. In SIGGRAPH '03, 2003.

[14] R. S. Laramee, H. Hauser, L. Zhao, and F. H. Post. Topology-Based Flow Visualization, The State of the Art. In Topology-Based Methods in Visualization Workshop, September 2005.

[15] R. S. Laramee, J. J. van Wijk, B. Jobard, and H. Hauser. ISA and IBFVS: Image Space-Based Visualization of Flow on Surfaces. IEEE Transactions on Visualization and Computer Graphics, 10(6):637-648, 2004.

[16] B. Lévy, S. Petitjean, N. Ray, and J. Maillot. Least Squares Conformal Maps for Automatic Texture Atlas Generation. In SIGGRAPH '02, pages 362-371, New York, NY, USA, 2002. ACM Press.

[17] W.-C. Li, B. Vallet, N. Ray, and B. Lévy. Representing Higher-Order Singularities in Vector Fields on Piecewise Linear Surfaces. IEEE Transactions on Visualization and Computer Graphics, 12(5):1315-1322, 2006.

[18] H. J. Lugt. Introduction to Vortex Theory. Vortex Flow Press, Inc., Potomac, Maryland, 1996.

[19] R. Peikert and M. Roth. The "Parallel Vectors" Operator - A Vector Field Visualization Primitive. In Proceedings of the conference on Visualization '99: celebrating ten years, pages $263-270,1999$.
[20] R. Peikert and F. Sadlo. Topology-guided Visualization of Constrained Vector Fields. In Topology-based Methods in Visualization, Mathematics+Visualization. Springer, 2007.

[21] N. Ray, W. C. Li, B. Lévy, A. Sheffer, and P. Alliez. Periodic Global Parameterization. ACM Trans. Graph., 25(4):1460-1485, 2006.

[22] L. Saboret, P. Alliez, and B. Lévy. Planar Parameterization of Triangulated Surface Meshes. In CGAL Editorial Board, editor, CGAL-3.2 User and Reference Manual. website, 2006. http://www.cgal.org/Manual/3.2/doc_html/cgal_manual/title.html.

[23] F. Sadlo, R. Peikert, and M. Sick. Visualization tools for vorticity transport analysis in incompressible flow. IEEE Transactions on Visualization and Computer Graphics, 12(5):949-956, 2006.

[24] G. Scheuermann and X. Tricoche. Topological methods in flow visualization. In C. Johnson and C. Hansen, editors, Visualization Handbook, pages 341-356. Academic Press, 2004.

[25] D. Sujudi and R. Haimes. Identification of Swirling Flow in 3-D Vector Fields. In 12th AIAA CFD Conference, San Diego CA, June 1995.

[26] A. Surana, O. Grunberg, and G. Haller. Exact theory of three-dimensional flow separation. Part I: Steady separation. J. Fluid Mech., 564:57-103, 2006.

[27] A. Surana, G. Jacobs, O. Grunberg, and G. Haller. Exact Theory of Three-Dimensional Flow Separation. Part II: Fixed Unsteady Separation. J. Fluid Mechanics, submitted, available at http://web.mit.edu/ghaller/www/papers.html, 2007.

[28] H. Theisel, J. Sahner, T. Weinkauf, H.-C. Hege, and H.-P. Seidel. Extraction of Parallel Vector Surfaces in 3D Time-Dependent Fields and Application to Vortex Core Line Tracking. In Proc. IEEE Visualization 2005, pages 631-638, Minneapolis, U.S.A., October 2005.

[29] H. Theisel and H.-P. Seidel. Feature Flow Fields. In VISSYM '03: Proceedings of the Symposium on Data Visualisation 2003, pages 141-148. Eurographics Association, 2003.

[30] X. Tricoche, T. Wischgoll, G. Scheuermann, and H. Hagen. Topology Tracking for the Visualization of Time-Dependent Two-Dimensional Flows. Computers \& Graphics, 26(2):249 - 257, 2002.

[31] K. Wang, Y. Tong, M. Desbrun, and P. Schröder. Edge Subdivision Schemes and the Construction of Smooth Vector Fields. ACM Transactions on Graphics (SIGGRAPH '06), 25(3):1041-1048, 2006.

[32] D. Weiskopf and T. Ertl. A Hybrid Physical/Device-Space Approach for Spatio-Temporally Coherent Interactive Texture Advection on Curved Surfaces. In Proc. of the 2004 Conference on Graphics Interface, pages 263-270. Canadian Human-Computer Communications Society, 2004.

[33] E. Zhang, K. Mischaikow, and G. Turk. Vector Field Design on Surfaces. ACM Trans. Graph., 25(4):1294-1326, 2006. 